Advances in Radio Science (2003) 1: 21-25

(C) Copernicus $\mathrm{GmbH} 2003$

\title{
Calibration of vector network analyzers on the basis of the LRR-method
}

\author{
I. Rolfes and B. Schiek
}

Institut für Hochfrequenztechnik, Ruhr-Universität Bochum, Universitätsstr. 150, 44801 Bochum, Germany

\begin{abstract}
The LRR method for the calibration of vector network analyzers is presented. This method belongs to the self calibration procedures where the calibration circuits might be partly unknown. The LRR calibration circuits are all of equal mechanical length in contrast to the well known TRL calibration, which needs a line-standard with a different length than the other calibration standards. For the LRR method it is thus not necessary to displace the connectors of the vector network analyzer during calibration in order to contact the calibration structures. The calibration circuits mainly consist of reflective networks that have to be placed at three consecutive positions. As the algorithm accounts for different distances between the reflective networks, the circuits are easy to realize. The robust functionality of the LRR method is confirmed by measurements.
\end{abstract}

\section{Introduction}

At microwave frequencies the complex scattering parameters of linear devices can be measured with vector network analyzers (VNA). The analyzers have to be calibrated in order to eliminate systematic errors from the measurement results. For the calibration of a VNA with four receivers it is advantageous to use self-calibration techniques where the calibration standards can be partly unknown. The commonly used TRL (Thru Reflect Line) method (Engen and Hoer, 1979; Eul and Schiek, 1991) needs a line-standard that differs in length from the other calibration standards. In order to contact the calibration circuits the distance between the connectors of the VNA has to be changed. Therefore a more complex testfixture is needed. The TRL method is thus for example not suited for applications where the connector distance is fixed.

The LRR method (Line Reflect) solves this problem, because its calibration circuits are all of equal mechanical length. This method can thus be used advantageously for instance in free space systems where a variation of the an-

Correspondence to: I. Rolfes (Ilona.Rolfes@ rub.de) tenna positions might be critical due to changes of the beam propagation. The calibration structures of the LRR method are very similar to the ones of the LNN method (Heuermann and Schiek, 1997). A symmetrical, reciprocal network, the so-called obstacle, is placed at three consecutive positions. While this obstacle network has to show a transmission for the LNN method, the networks of the LRR method consist of reflections, which might or might not show a transmission. The LRR method is thus able to perform a calibration on the basis of reflective networks, which leads to an enlargement of the bandwidth in comparison to the LNN method. The calibration structures of the LRR method can be realized very easily as etched structures in microstrip technology or as metal plates for free space applications for instance.

In the following the theory of the LRR method is presented, where in principal two cases are distinguished. In the first one, the obstacles are assumed to show no transmission at all and in the second one, the obstacles might or might not show a weak transmission. Both solutions are described, because depending on the realized calibration structures the appropriate way should be chosen in order to improve the accuracy. In addition the algorithm of the LRR method for different distances between the obstacle positions is presented.

\section{The LRR method}

The calibration circuits of the LRR method consist of two line-elements of the mechanical length $l$ and of an object to be calibrated, the so-called obstacle, which has to be placed at three consecutive positions as depicted in Fig. 1, comparable to the LNN calibration. The main difference to the LNN method is that the LRR obstacle networks may be transmission free. A description of the obstacle networks on the basis of transmission matrices as for the LNN method is thus not possible. At first the LRR method is presented for the case that the obstacles show no transmission at all. 

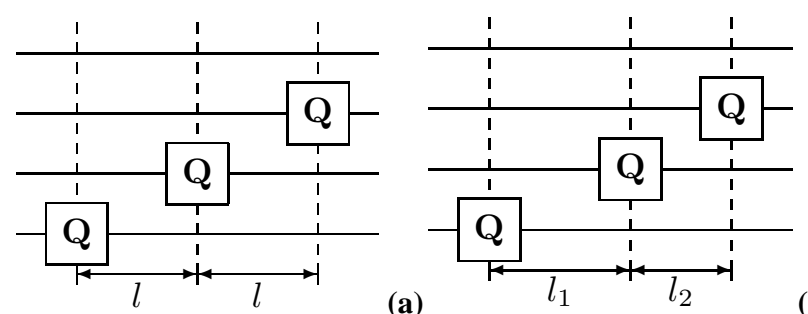

(b)

Fig. 1. Principal setup of the LRR calibration circuits with (a) equal and (b) different lengths.

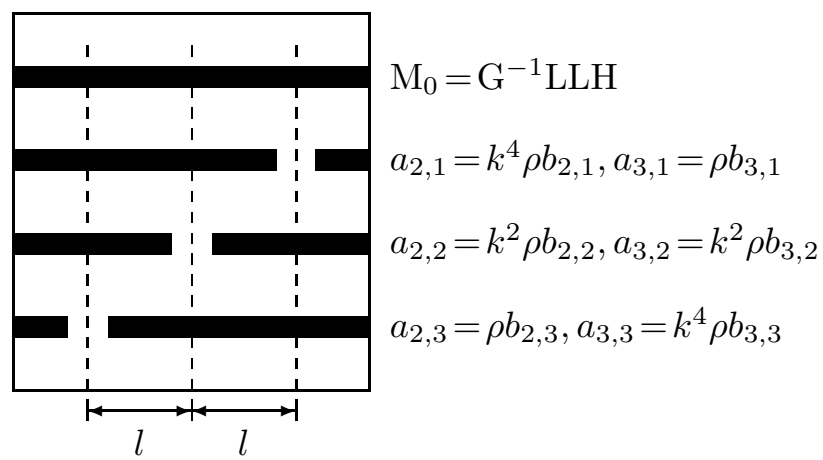

Fig. 2. LRR calibration structures in microstrip technology.

\subsection{LRR method without transmission}

For this variant the calibration structures may be realized e.g. in microstrip technology as open-ended transmission lines as depicted in Fig. 2. The calibration circuits can be described with the help of a line parameter $k=e^{-\gamma l}$ with the unknown propagation constant $\gamma$ and a reflection coefficient $\rho$. The wave parameters $a_{1, i}, \ldots, a_{4, i}$ and $b_{1, i} \ldots b_{4, i}, i=1 \ldots 3$ are defined according to the setup in Fig. 3 where the reflection coefficients $\rho_{l, i}$ and $\rho_{r, i}$ refer to the $i$ different positions of the reflective networks. $\mathbf{G}$ and $\mathbf{H}$ are two-ports which represent the systematic errors of the VNA. During the selfcalibration $\mathbf{G}$ and $\mathbf{H}$ are eliminated in order to determine the unknown line parameter $k$ and the reflection coefficient $\rho$.

For this purpose the error two-port $\mathbf{G}^{-1}$ is described by the following equation with $\tilde{\mathbf{G}}=\mathbf{G}^{-1}$ :

$\left[\begin{array}{c}b_{1, i} \\ a_{1, i}\end{array}\right]=\tilde{\mathbf{G}}\left[\begin{array}{l}a_{2, i} \\ b_{2, i}\end{array}\right]=\tilde{\mathbf{G}}\left[\begin{array}{c}\rho_{l, i} b_{2, i} \\ b_{2, i}\end{array}\right]$

resulting in a bilinear transformation (Schiek and Gronefeld, 1999).

$\nu_{l, i}=\frac{b_{1, i}}{a_{1, i}}=\frac{\tilde{G}_{11} \rho_{l, i} b_{2, i}+\tilde{G}_{12} b_{2, i}}{\tilde{G}_{21} \rho_{l, i} b_{2, i}+\tilde{G}_{22} b_{2, i}}=\frac{\tilde{G}_{11} \rho_{l, i}+\tilde{G}_{12}}{\tilde{G}_{21} \rho_{l, i}+\tilde{G}_{22}}$

Such a bilinear transformation, also known as Möbiustransformation, is generally defined as, (Schiek, 1999),

$x_{j}=\frac{C_{1} y_{j}+C_{2}}{C_{3} y_{j}+C_{4}}$

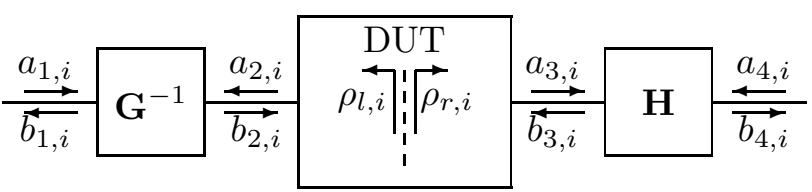

Fig. 3. Simplified block diagramm of the analyzer setup

where the two variables $x_{j}$ and $y_{j}$ correspond to the measurement value $v_{l, i}$ and the unknown calibration standard parameter $\rho_{l, i}$ and the constants $C_{1}, \ldots, C_{4}$ represent the error two port parameters of Eq. (2). Concerning the two-port $\mathrm{H}$ a similar equation can be found on the basis of the wave parameter description in Fig. 3.

$\left[\begin{array}{l}a_{4, i} \\ b_{4, i}\end{array}\right]=\mathbf{H}^{-1}\left[\begin{array}{l}b_{3, i} \\ a_{3, i}\end{array}\right]=\mathbf{H}^{-1}\left[\begin{array}{c}b_{3, i} \\ \rho_{r, i} b_{3, i}\end{array}\right]$

With the help of the first structure of the LRR method in Fig. 2, the through connection with $\mathbf{M}_{\mathbf{0}}=\mathbf{G}^{-1} \mathbf{L L H}$, the dependence of the error two port parameter $\mathbf{H}$ can be replaced by the error parameter $\tilde{\mathbf{G}}$ :

$\mathbf{M}_{\mathbf{0}}\left[\begin{array}{l}a_{4, i} \\ b_{4, i}\end{array}\right]=\left[\begin{array}{c}a_{4, i}^{\prime} \\ b_{4, i}^{\prime}\end{array}\right]=\mathbf{G}^{-1} \mathbf{L} \mathbf{L}\left[\begin{array}{c}b_{3, i} \\ \rho_{r, i} b_{3, i}\end{array}\right]=$

$\tilde{\mathbf{G}}\left[\begin{array}{c}k^{2} b_{3, i} \\ \rho_{r, i} k^{-2} b_{3, i}\end{array}\right]$ with $\quad \mathbf{L}=\left[\begin{array}{cc}k & 0 \\ 0 & k^{-1}\end{array}\right]$

In this way, another bilinear transformation in the error two port parameter $\tilde{\mathbf{G}}$ results with $\tilde{\rho}_{r, i}=k^{4} \rho_{r, i}^{-1}$.

$v_{r, i}=\frac{a_{4, i}^{\prime}}{b_{4, i}^{\prime}}=\frac{\tilde{G}_{11} k^{2} b_{3, i}+\tilde{G}_{12} \rho_{r, i} k^{-2} b_{3, i}}{\tilde{G}_{21} k^{2} b_{3, i}+\tilde{G}_{22} \rho_{r, i} k^{-2} b_{3, i}}=$
$\frac{\tilde{G}_{11} k^{4} \rho_{r, i}^{-1}+\tilde{G}_{12}}{\tilde{G}_{21} k^{4} \rho_{r, i}^{-1}+\tilde{G}_{22}}=\frac{\tilde{G}_{11} \tilde{\rho}_{r, i}+\tilde{G}_{12}}{\tilde{G}_{21} \tilde{\rho}_{r, i}+\tilde{G}_{22}}$

The reflection coefficients $\rho_{l, i}$ and $\tilde{\rho}_{r, i}$ correspond to the measurement values $v_{l, i}$ and $v_{r, i}$ with:

$\rho_{l, 1}=k^{4} \rho \Rightarrow v_{l, 1}, \tilde{\rho}_{r, 1}=k^{4} \rho^{-1} \Rightarrow v_{r, 1}$
$\rho_{l, 2}=k^{2} \rho \Rightarrow v_{l, 2}, \tilde{\rho}_{r, 2}=k^{2} \rho^{-1} \Rightarrow v_{r, 2}$
$\rho_{l, 3}=\rho \quad \Rightarrow v_{l, 3}, \tilde{\rho}_{r, 3}=\rho^{-1} \Rightarrow v_{r, 3}$

On the basis of the measurement of four reflection coefficients four equations of the type of Eqs. (2) and (6) result, so that the unknown error two port parameters $\tilde{G}_{11}, \tilde{G}_{12}, \tilde{G}_{21}$ and $\tilde{G}_{22}$ can be eliminated. This can be performed with the help of the cross ratio

$\frac{\left(y_{1}-y_{2}\right)\left(y_{3}-y_{4}\right)}{\left(y_{1}-y_{4}\right)\left(y_{3}-y_{2}\right)}=\frac{\left(x_{1}-x_{2}\right)\left(x_{3}-x_{4}\right)}{\left(x_{1}-x_{4}\right)\left(x_{3}-x_{2}\right)}$

which generally holds for a bilinear transformation as given in Eq. (3). A set of equations can thus be constructed, which only depends on the unknown reflection coefficient $\rho$ and the unknown line parameter $k$ in dependence of the measurement values $v_{l, i}$ and $v_{r, i}$, as e.g.: 


$$
\begin{aligned}
\tilde{v}_{1}= & \frac{\left(v_{l, 3}-v_{r, 3}\right)\left(v_{r, 2}-v_{l, 2}\right)}{\left(v_{l, 3}-v_{l, 2}\right)\left(v_{r, 2}-v_{r, 3}\right)} \\
= & \frac{\left(\rho-\rho^{-1}\right)\left(k^{2} \rho^{-1}-k^{2} \rho\right)}{\left(\rho-k^{2} \rho\right)\left(k^{2} \rho^{-1}-\rho^{-1}\right)} \\
\tilde{v}_{2}= & \frac{\left(v_{l, 3}-v_{l, 1}\right)\left(v_{r, 1}-v_{r, 3}\right)}{\left(v_{l, 3}-v_{r, 3}\right)\left(v_{r, 1}-v_{l, 1}\right)} \\
& =\frac{\left(\rho-k^{4} \rho\right)\left(k^{4} \rho^{-1}-\rho^{-1}\right)}{\left(\rho-\rho^{-1}\right)\left(k^{4} \rho^{-1}-k^{4} \rho\right)} \\
\tilde{v}_{3}= & \frac{\left(v_{l, 3}-v_{l, 1}\right)\left(v_{r, 3}-v_{r, 2}\right)}{\left(v_{l, 3}-v_{r, 2}\right)\left(v_{r, 3}-v_{l, 1}\right)} \\
& =\frac{\left(\rho-k^{4} \rho\right)\left(\rho^{-1}-k^{2} \rho\right)}{\left(\rho-k^{2} \rho\right)\left(\rho^{-1}-k^{4} \rho\right)}
\end{aligned}
$$

The line parameter and the reflection coefficient are thus calculable as follows:

$k^{4}+k^{2}\left(2-\tilde{v}_{1} \tilde{v}_{2}\right)+1=0, \quad \rho^{2}=\frac{\tilde{v}_{3}-1-k^{2}}{k^{2}\left(k^{2}\left(\tilde{v}_{3}-1\right)-1\right)}$

2.2 LRR method without transmission and unequal obstacle distances

In order to account for different lengths between the obstacle positions as shown in Fig. $1 \mathrm{~b}$ the following algorithm has to be considered. The line elements of the mechanical lengths $l_{1}$ and $l_{2}$ can be described in dependence of the propagation constant $\gamma$ with the line parameters $k_{1}=e^{-\gamma l_{1}}$ and $k_{2}=$ $e^{-\gamma l_{2}}$ and the corresponding transmission matrices $\mathbf{L}_{\mathbf{1}}$ and $\mathbf{L}_{2}$. According to Fig. 3 the following wave parameters can be defined:

$$
\begin{array}{ll}
a_{2,1}=k_{1}^{2} k_{2}^{2} \rho b_{2,1}, & a_{3,1}=\rho b_{3,1} \\
a_{2,2}=k_{1}^{2} \rho b_{2,2}, & a_{3,2}=k_{2}^{2} \rho b_{3,2} \\
a_{2,3}=\rho b_{2,3}, & a_{3,3}=k_{1}^{2} k_{2}^{2} \rho b_{3,3}
\end{array}
$$

Considering the previous description of the error two-port G, Eq. (2) is also valid for this calibration setup. On the other hand for the error two port $\mathbf{H}$ a modified relation results. Starting from Eq. (4) and with $\mathbf{H}^{-1}=\mathbf{M}_{\mathbf{0}}{ }^{-1} \mathbf{G}^{-1} \mathbf{L}_{\mathbf{1}} \mathbf{L}_{\mathbf{2}}$ one gets:

$\mathbf{M}_{\mathbf{0}}\left[\begin{array}{l}a_{4} \\ b_{4}\end{array}\right]=\left[\begin{array}{l}a_{4}^{\prime} \\ b_{4}^{\prime}\end{array}\right]=\mathbf{G}^{-1} \mathbf{L}_{\mathbf{1}} \mathbf{L}_{\mathbf{2}}\left[\begin{array}{c}b_{3, i} \\ \rho_{r, i} b_{3, i}\end{array}\right]=$

$\tilde{\mathbf{G}}\left[\begin{array}{c}k_{1} k_{2} b_{3, i} \\ \rho_{r, i} k_{1}^{-1} k_{2}^{-1} b_{3, i}\end{array}\right]$,

so that the following bilinear relation results

$$
v_{r, i}=\frac{a_{4, i}^{\prime}}{b_{4, i}^{\prime}}=\frac{\tilde{G}_{11} k_{1}^{2} k_{2}^{2} \rho_{r, i}^{-1}+\tilde{G}_{12}}{\tilde{G}_{21} k_{1}^{2} k_{2}^{2} \rho_{r, i}^{-1}+\tilde{G}_{22}}=\frac{\tilde{G}_{11} \tilde{\rho}_{r, i}+\tilde{G}_{12}}{\tilde{G}_{21} \tilde{\rho}_{r, i}+\tilde{G}_{22}} .
$$

For the structures depicted in Fig. 1b with:

$$
\begin{aligned}
& \rho_{l, 1}=k_{1}^{2} k_{2}^{2} \rho \Rightarrow v_{l, 1}, \quad \tilde{\rho}_{r, 1}=k_{1}^{2} k_{2}^{2} \rho^{-1} \Rightarrow v_{r, 1} \\
& \rho_{l, 2}=k_{1}^{2} \rho \Rightarrow v_{l, 2}, \quad \tilde{\rho}_{r, 2}=k_{1}^{2} \rho^{-1} \Rightarrow v_{r, 2} \\
& \rho_{l, 3}=\rho \Rightarrow v_{l, 3}, \quad \tilde{\rho}_{r, 3}=\rho^{-1} \Rightarrow v_{r, 3}
\end{aligned}
$$

different bilinear relations can be written.

$$
\begin{aligned}
\tilde{v}_{1}= & \frac{\left(v_{l, 3}-v_{r, 3}\right)\left(v_{r, 2}-v_{l, 2}\right)}{\left(v_{l, 3}-v_{l, 2}\right)\left(v_{r, 2}-v_{r, 3}\right)}= \\
& \frac{\left(\rho-\rho^{-1}\right)\left(k_{1}^{2} \rho^{-1}-k_{1}^{2} \rho\right)}{\left(\rho-k_{1}^{2} \rho\right)\left(k_{1}^{2} \rho^{-1}-\rho^{-1}\right)} \\
\tilde{v}_{2}= & \frac{\left(v_{l, 3}-v_{l, 1}\right)\left(v_{r, 1}-v_{r, 3}\right)}{\left(v_{l, 3}-v_{r, 3}\right)\left(v_{r, 1}-v_{l, 1}\right)}= \\
& \frac{\left(\rho-k_{1}^{2} k_{2}^{2} \rho\right)\left(k_{1}^{2} k_{2}^{2} \rho^{-1}-\rho^{-1}\right)}{\left(\rho-\rho^{-1}\right)\left(k_{1}^{2} k_{2}^{2} \rho^{-1}-k_{1}^{2} k_{2}^{2} \rho\right)} \\
\tilde{v}_{3}= & \frac{\left(v_{l, 1}-v_{l, 2}\right)\left(v_{r, 2}-v_{r, 1}\right)}{\left(v_{l, 1}-v_{r, 1}\right)\left(v_{r, 2}-v_{l, 2}\right)}= \\
& \frac{\left(k_{1}^{2} k_{2}^{2} \rho-k_{1}^{2} \rho\right)\left(k_{1}^{2} \rho^{-1}-k_{1}^{2} k_{2}^{2} \rho^{-1}\right)}{\left(k_{1}^{2} k_{2}^{2} \rho-k_{1}^{2} k_{2}^{2} \rho^{-1}\right)\left(k_{1}^{2} \rho^{-1}-k_{1}^{2} \rho\right)} \\
\tilde{v}_{4}= & \frac{\left(v_{l, 3}-v_{l, 1}\right)\left(v_{r, 3}-v_{l, 2}\right)}{\left(v_{l, 3}-v_{l, 2}\right)\left(v_{r, 3}-v_{l, 1}\right)}= \\
& \frac{\left(\rho-k_{1}^{2} k_{2}^{2} \rho\right)\left(\rho^{-1}-k_{1}^{2} \rho\right)}{\left(\rho-k_{1}^{2} \rho\right)\left(\rho^{-1}-k_{1}^{2} k_{2}^{2} \rho\right)}
\end{aligned}
$$

After some transformations the following equations for the determination of the line parameters and the reflection coefficient result:

$$
\begin{aligned}
& k_{2}^{2} \pm k_{2} \frac{\tilde{v}_{1} \tilde{v}_{3}-\tilde{v}_{1} \tilde{\nu}_{2}-1}{\sqrt{\tilde{\nu}_{1} \tilde{v}_{2}}}+1=0, \quad k_{1}=\frac{ \pm \sqrt{\tilde{v}_{1} \tilde{v}_{3}}}{ \pm \sqrt{\tilde{v}_{1} \tilde{v}_{2}}-k_{2}}, \\
& \rho^{2}=\frac{1}{k_{1}^{2}} \cdot \frac{1-k_{1}^{2} k_{2}^{2}+\tilde{v}_{4}\left(k_{1}^{2}-1\right)}{1-k_{1}^{2} k_{2}^{2}+\tilde{v}_{4} k_{2}^{2}\left(k_{1}^{2}-1\right)}
\end{aligned}
$$

The two previously discussed solutions are based on the premise that the obstacle networks show no transmission. For the case that they may have a weak transmission, the following variant of the LRR method is appropriate.

\subsection{LRR method with a weak transmission}

This algorithm is based on the representation of the obstacle networks with pseudo-transmission matrices. As the obstacles might also be realized as pure reflections, the networks cannot be described with transmission matrices, because in this representation a factor $\Delta m_{i}$ might become zero. According to Fig. 3 the measurement matrix is defined as follows,

$\mathbf{M}_{\mathbf{i}}=\left[\begin{array}{ll}b_{1, i}^{\prime} & b_{1, i}^{\prime \prime} \\ a_{1, i}^{\prime} & a_{1, i}^{\prime \prime}\end{array}\right] \frac{1}{a_{4, i}^{\prime} b_{4, i}^{\prime \prime}-a_{4, i}^{\prime \prime} b_{4, i}^{\prime}}\left[\begin{array}{cc}b_{4, i}^{\prime \prime} & -a_{4, i}^{\prime \prime} \\ -b_{4, i}^{\prime} & a_{4, i}^{\prime}\end{array}\right]$

where the primes indicate from which side of the setup the generator signal is fed in. By multiplying the measurement matrices with the factor $\Delta m_{i}=a_{4, i}^{\prime} b_{4, i}^{\prime \prime}-a_{4, i}^{\prime \prime} b_{4, i}^{\prime}$ the pseudotransmission matrices $\tilde{\mathbf{Q}}_{\mathbf{i}}$ result.

$$
\begin{aligned}
\tilde{\mathbf{Q}}_{\mathbf{i}}= & \frac{\Delta m_{i}}{S_{21, i}}\left[\begin{array}{cc}
S_{12, i} S_{21, i}-S_{11, i}^{2} & S_{11, i} \\
-S_{11, i} & 1
\end{array}\right]= \\
& \frac{1}{\mu_{f, i}}\left[\begin{array}{cr}
\mu_{f, i} \mu_{r, i}-\rho^{2} & \rho \\
-\rho & 1
\end{array}\right]
\end{aligned}
$$


The transmission characteristics in forward and reverse direction are described by $\mu_{f, i}$ and $\mu_{r, i}$. These values are related to the scattering parameters of the obstacle as follows: $\mu_{f, i}=S_{21, i} / \Delta m_{i}, \mu_{r, i}=S_{12, i} \cdot \Delta m_{i}$ and $\mu_{f, i} \cdot \Delta m_{i}=$ $\Delta m_{i} / \mu_{r, i}$ taking into account the reciprocity of the obstacle. In order to determine the unknown obstacle and line parameters the obstacle networks are positioned as depicted in Fig. 2. Considering the setup in Fig. 1a, the following pseudo-transmission matrices can be constructed with $\tilde{\mathbf{M}}_{\mathbf{i}}=\Delta m_{i} \mathbf{M}_{\mathbf{i}}$ and $\tilde{\mathbf{Q}}_{\mathbf{i}}=\Delta m_{i} \mathbf{Q}$ for $i=1, \ldots, 3$ :

$\tilde{\mathbf{M}}_{\mathbf{1}}=\mathbf{G}^{-1} \tilde{\mathbf{Q}}_{\mathbf{1}} \mathbf{L L H}$,

$\tilde{\mathbf{M}}_{\mathbf{2}}=\mathbf{G}^{-1} \mathbf{L} \tilde{\mathbf{Q}}_{\mathbf{2}} \mathbf{L H}$,

$\tilde{\mathbf{M}}_{\mathbf{3}}=\mathbf{G}^{-1} \mathbf{L} \mathbf{L} \tilde{\mathbf{Q}}_{\mathbf{3}} \mathbf{H}$

For the obstacle matrices at the different positions it can be found that $\tilde{\mathbf{Q}}_{\mathbf{1}}=\hat{\mathbf{Q}} / \mu_{f 1}, \tilde{\mathbf{Q}} \mathbf{2}=\hat{\mathbf{Q}} / \mu_{f 2}$ and $\tilde{\mathbf{Q}} \mathbf{3}=\hat{\mathbf{Q}} / \mu_{f 3}$ where $\hat{\mathbf{Q}}$ is defined as:

$\hat{\mathbf{Q}}=\left[\begin{array}{cc}\mu_{f 1} \mu_{r 1}-\rho^{2} & \rho \\ -\rho & 1\end{array}\right]=\left[\begin{array}{ll}\hat{q}_{11} & \hat{q}_{12} \\ \hat{q}_{21} & \hat{q}_{22}\end{array}\right]$

with $\mu_{f 1} \mu_{r 1}=\mu_{f 2} \mu_{r 2}=\mu_{f 3} \mu_{r 3}$ because of the reciprocity.

On the basis of this representation, the following trace relations can be evaluated:

$$
\begin{aligned}
& \delta_{1}=\operatorname{tr}\left\{\tilde{\mathbf{M}}_{\mathbf{1}} \mathbf{M}_{\mathbf{0}}{ }^{-1}\right\}=\mu_{f 1}^{-1} \operatorname{tr}\{\hat{\mathbf{Q}}\} \\
& \delta_{2}=\operatorname{tr}\left\{\tilde{\mathbf{M}}_{\mathbf{2}} \mathbf{M}_{\mathbf{0}}{ }^{-1}\right\}=\mu_{f 2}^{-1} \operatorname{tr}\{\hat{\mathbf{Q}}\} \\
& \delta_{3}=\operatorname{tr}\left\{\tilde{\mathbf{M}}_{\mathbf{3}} \mathbf{M}_{\mathbf{0}}{ }^{-1}\right\}=\mu_{f 3}^{-1} \operatorname{tr}\{\hat{\mathbf{Q}}\} \\
& \delta_{4}=\operatorname{tr}\left\{\tilde{\mathbf{M}}_{\mathbf{2}} \mathbf{M}_{\mathbf{0}}{ }^{-1} \tilde{\mathbf{M}}_{\mathbf{1}} \mathbf{M}_{\mathbf{0}}{ }^{-1}\right\} \\
& =\mu_{f 1}^{-1} \mu_{f 2}^{-1} \operatorname{tr}\left\{\hat{\mathbf{Q}} \mathbf{L} \hat{\mathbf{Q}} \mathbf{L}^{-1}\right\} \\
& \delta_{6}=\operatorname{tr}\left\{\tilde{\mathbf{M}}_{3} \mathbf{M}_{\mathbf{0}}{ }^{-1} \tilde{\mathbf{M}}_{\mathbf{1}} \mathbf{M}_{\mathbf{0}}{ }^{-1}\right\} \\
& =\mu_{f 1}^{-1} \mu_{f 3}^{-1} \operatorname{tr}\left\{\hat{\mathbf{Q}} \mathbf{L} \mathbf{L} \hat{\mathbf{Q}} \mathbf{L}^{-1} \mathbf{L}^{-1}\right\}
\end{aligned}
$$

These equations can be transformed into a set of nonlinear equations.

$$
\begin{aligned}
\mu_{f 1} \delta_{1} & =\hat{q}_{11}+\hat{q}_{22} \\
\mu_{f 1} \mu_{f 2} \delta_{4} & =\hat{q}_{11}^{2}+\hat{q}_{22}^{2}+\hat{q}_{12} \hat{q}_{21}\left(k^{2}+k^{-2}\right) \\
\mu_{f 1} \mu_{f 3} \delta_{6} & =\hat{q}_{11}^{2}+\hat{q}_{22}^{2}+\hat{q}_{12} \hat{q}_{21}\left(k^{4}+k^{-4}\right)
\end{aligned}
$$

The unknown parameters can thus be determined as follows

$$
\begin{aligned}
& \left(k+k^{-1}\right)^{2}=\frac{\delta_{1} \delta_{6} \delta_{3}^{-1}-\delta_{1}^{2}+2 \Delta m_{1}^{2}}{\delta_{1} \delta_{4} \delta_{2}^{-1}-\delta_{1}^{2}+2 \Delta m_{1}^{2}}, \\
& \mu_{f 1}=\frac{1}{2 h_{1}} \cdot\left(-h_{2} \pm \sqrt{h_{2}^{2}-4 h_{3}}\right), \\
& \rho^{2}=\Delta m_{1}^{2} \mu_{f 1}^{2}-\mu_{f 1} \delta_{1}+1
\end{aligned}
$$

with

$$
\begin{aligned}
& h_{1}=\delta_{1} \delta_{4} \delta_{2}^{-1}-\delta_{1}^{2}+\Delta m_{1}^{2}\left(2+h_{3}\right), \\
& h_{2}=-\delta_{1} h_{3} \\
& h_{3}=\left(k-k^{-1}\right)^{2} .
\end{aligned}
$$

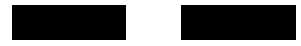

(a)

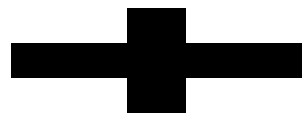

(b)
Fig. 4. Obstacle networks (a) without or (b) with transmission.

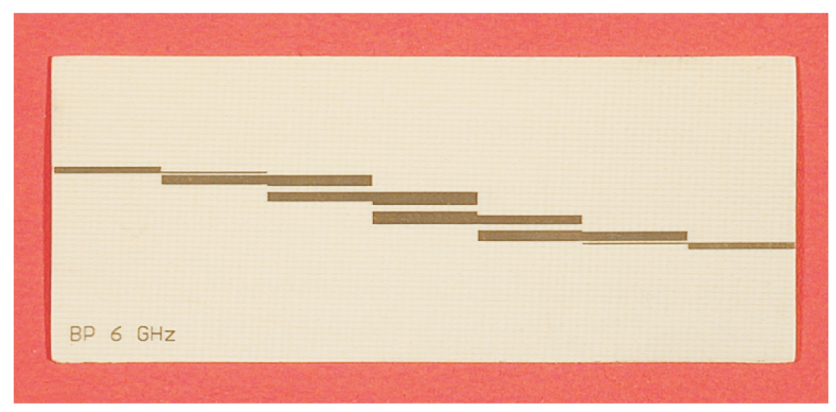

Fig. 5. Foto of the device under test which is a bandpass filter.

As already explained in conjunction with the other methods, an approximate knowledge of the circuits dimensions is necessary in order to choose the correct solutions.

\section{Experimental results}

For the verification of the developed LRR method the calibration circuits are realized in microstrip technology.

The obstacles consist of open ended transmission lines as depicted in Figs. 2 and 4. They might also be realized as open ended stubs as shown in Fig. 4. The LRR solution reveals singularities when the electrical length of the line element becomes a multiple of the half wavelength, as is also known from the TRL and the LNN methods.

Measurements of a planar stripline bandpass filter as shown in Fig. 5 were performed with a VNA (HP8510C) on the basis of the LRR method in comparison to the TRL method. The measurements lead to similar results which are shown in Fig. 6. The measured scattering parameters show a good agreement between the two methods.

\section{Conclusion}

A new self calibration procedure, the LRR method, is presented. The LRR calibration circuits are all of equal mechanical length. This is advantageous for the calibration of vector network analyzers, as for instance for applications where the connectors of the analyzer measurement ports cannot be displaced. A realization of the calibration circuits as etched structures in microstrip technology is shown. The precision of the LRR method does not depend on the precise positioning of the obstacle networks, because the algorithm accounts for nonequal distances between the obstacles. The robust functionality of the LRR method is confirmed by measurements. 

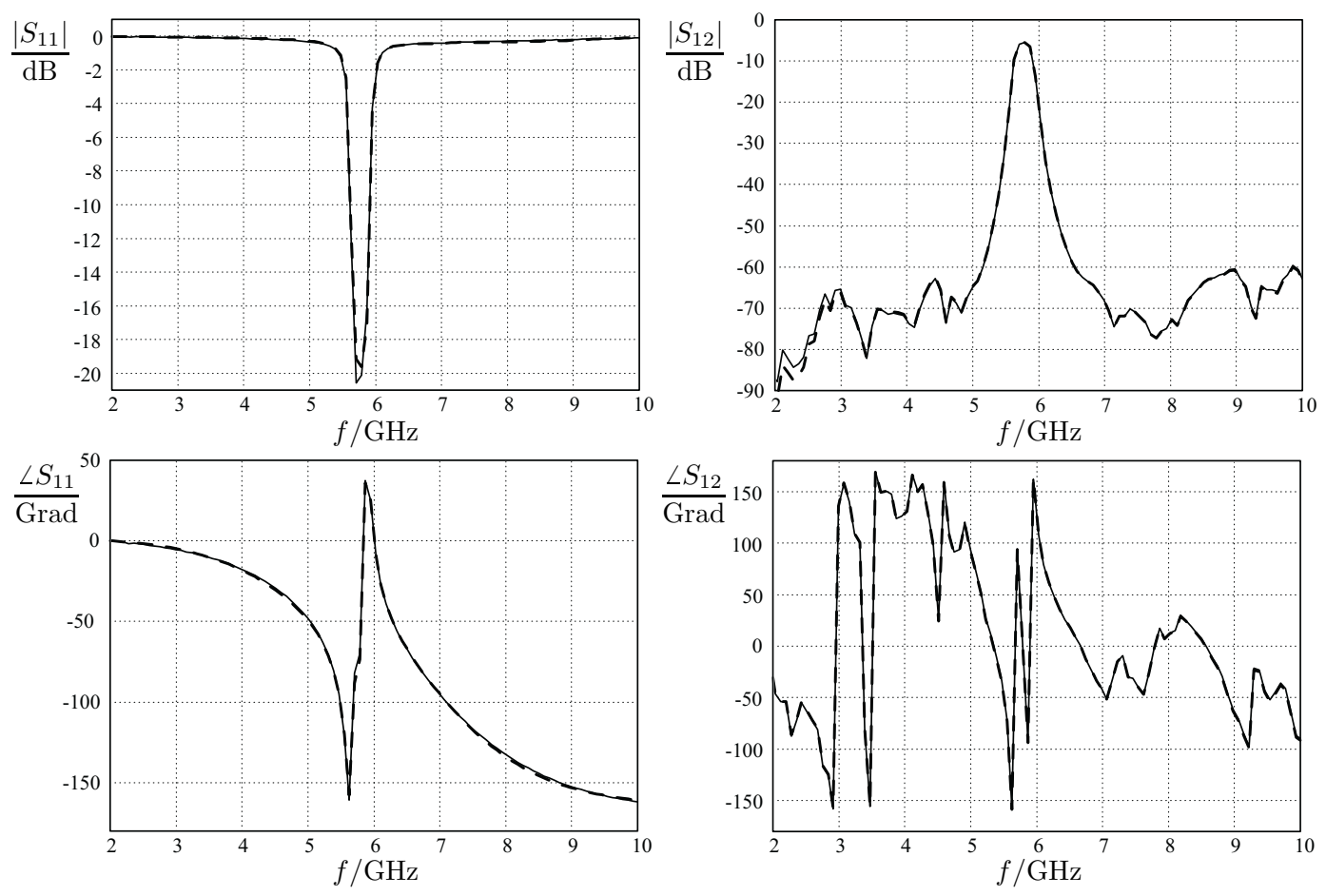

Fig. 6. TRL (- -) and LRR method (一) applied to a bandpass-filter.

\section{References}

Engen, G. F. and Hoer, C. A.: Thru-Reflect-Line: An improved technique for calibrating the dual six port automatic network analyzer, IEEE Trans. Microw. Theory Tech., 27, pp. 987-993, Dec. 1979.

Eul, H.-J. and Schiek, B.: A Generalized Theory and New Calibration Procedures for Network Analyzer Self-Calibration, IEEE Trans. Microw. Theory Tech., 39, pp. 724-731, April 1991.

Eul, H.-J. and Schiek, B.: Thru-Match-Reflect: One result of a rigorous theory for deembedding and network analyzer calibration, Proc. 18th EUMC, pp. 909-914, 1988.

Williams, D. F. and Marks, R. B.: LRM Probe-Tip Calibrations using Nonideal Standards, IEEE Trans. Microw. Theory Tech., 43, pp. 466-469, Feb. 1995.

Heuermann, H. and Schiek, B.: Line Network Network (LNN): An Alternative In-Fixture Calibration Procedure, IEEE Trans. Microw. Theory Tech., 45, pp. 408-413, March 1997.

Schiek, B.: Grundlagen der Hochfrequenz-Messtechnik, SpringerVerlag Berlin, pp. 173-174, 1999.

Schiek, B. and Gronefeld, A.: Standing Wave Meters and Network Analyzers, Wiley Encyclopedia of Electrical and Electronics Engineering, 20, pp. 403-423, 1999. 